\title{
Discussion: Radial Strain Behaviors and Stress State Interpretation of Soil under Direct Simple Shear
}

Li, Y ${ }^{1}$, Yang, Y. M ${ }^{2}$., Roberts, G. $W^{3}$., Yu, H. S ${ }^{4}$.

${ }_{1} \mathrm{PhD}$ Candidate, International Doctoral Innovation Centre, The University of Nottingham

Ningbo China, 199 Taikang East Road, Ningbo, China. E-mail: Yao.Li@ nottingham.edu.cn.

2 Associate Professor, International Doctoral Innovation Centre, The University of Nottingham Ningbo China, 199 Taikang East Road, Ningbo, China. Tel: +86 (0)574 88182407. Fax: +86 (0)574 88180175. E-mail: Ming.yang@ nottingham.edu.cn.

${ }_{3}^{3}$ Professor, Department of Civil Engineering, The University of Nottingham Ningbo China, 199 Taikang East Road, Ningbo, China. E-mail: Gethin.roberts@ nottingham.edu.cn.

4 Professor, Faculty of Engineering, The University of Nottingham, University Park, Nottingham, UK. E-mail: hai-sui.yu@nottingham.ac.uk.

\section{The discussed paper:}

Kang, Xin, Cheng, Yao, and Ge, Louis, "Radial Strain Behaviors and Stress State Interpretation of Soil Under Direct Simple Shear," Journal of Testing and Evaluation, Vol. 43, No. 6, 2015, pp. 15941601, doi:10.1520/JTE20140202. ISSN 0090-3973 


\section{Abstract}

Two methods are used in determining the stress state of simple shear tests in the discussed paper. The authors state that the second method is proposed by Oda and Konishi, based on the distribution law of contact force [1]. However, the relation used in the method is found by Roscoe, Bassett and Cole from experimental results [2]. In addition, the determination of the constant $\mathrm{k}$, which uses $k=1-K_{0}$, is problematic in the discussed paper. First, the equation can only be deduced after some assumptions are made. Second, the value of $\mathrm{k}$ will not be a constant if the $\mathrm{K}_{0}$ changes.

\section{Keywords}

Direct simple shear, Stress state interpretation, $\mathrm{K}_{0}$, Shear behavior 
The authors present an interesting paper on radial strain behaviors of soil under direct simple shear. Two methods are used in interpreting the stress state of NGI-type simple shear tests, which are "pure shear method" and "a method proposed by Oda and Konishi [1]". The second method is based on the Equation 5 in the discussed paper: $\frac{\tau}{\sigma_{v c}}=k \tan \psi$. The authors describe the equation only as an assumption made by Oda and Konishi [1]. However, this equation is already proposed by Roscoe, Bassett and Cole [2], based on experimental results using Cambridge-type simple shear apparatus. This relation is then validated by other researchers [3,4]. Fig. 1 shows the linear relation between $\frac{\tau}{\sigma_{v c}}$ and $\tan \psi$. The figure includes results from constant load tests on Leighton Buzzard sand at different stress levels and relative densities, with different stress or strain paths [5].

FIG. 1 Relation between $\frac{\tau}{\sigma_{v c}}$ and $\tan \psi$ in simple shear tests [3,4].

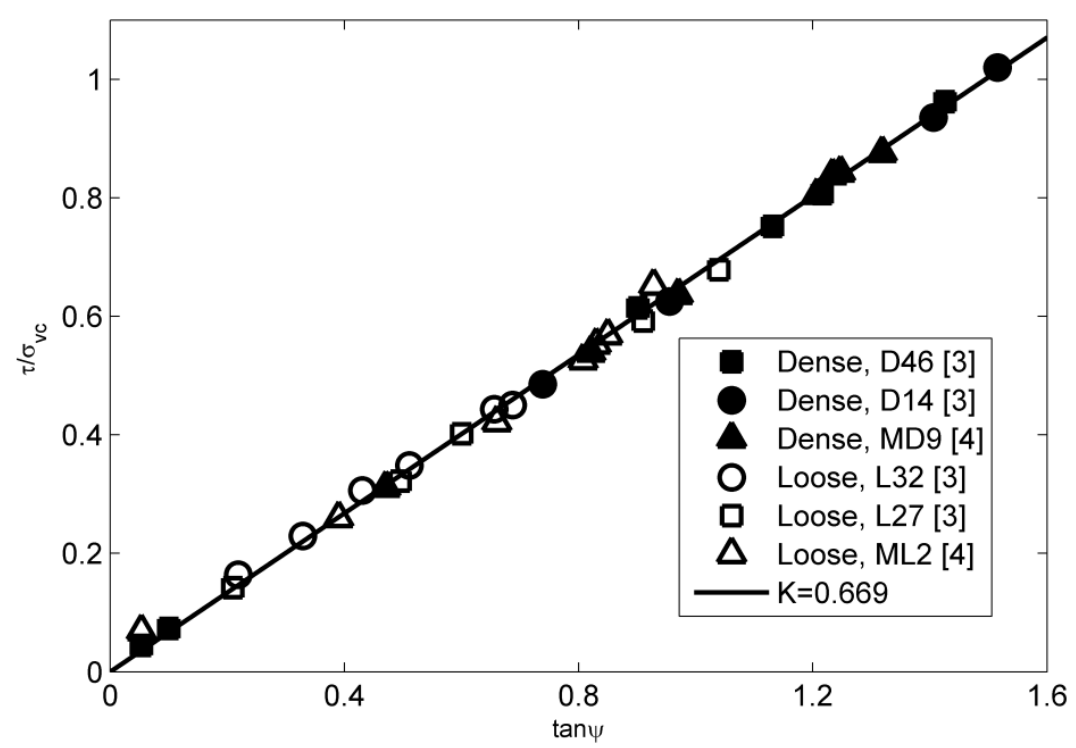

The physical meaning of the constant $\mathrm{k}$ is not clear [4], and it is independent of void ratio, as shown in Fig. 1. As a result, the value of $\mathrm{k}$ value should be determined carefully for different materials. In the second interpretation method, the authors used an equation: $k=1-K_{0}=\sin \phi_{u}$, to calculate the value of $\mathrm{k}$. However, this equation can only be deduced if some assumptions are made. 
Stress ratio, $\frac{t}{s}=\frac{\sigma_{1}-\sigma_{3}}{\sigma_{1}+\sigma_{3}}$, can be obtained by inserting Equation 7 and Equation 8 in the discussed paper, which is

$$
\frac{t}{s}=\frac{\left(\frac{\tau}{\sigma_{v}}\right)^{2}+k^{2}}{\left(\frac{\tau}{\sigma_{v}}\right)^{2}+k(2-\mathrm{k})}
$$

At the start of shearing, $\frac{\tau}{\sigma_{v}}=0$ and samples are at a $K_{0}$ consolidation $\left(\frac{t}{s}=\frac{1-k_{0}}{1+k_{0}}\right)$, which gives

$$
\frac{1-k_{0}}{1+k_{0}}=\frac{k^{2}}{k(2-\mathrm{k})} \text {. }
$$

Solving the equation, $k=1-K_{0}$. This relation is built on the assumptions that the sample is at the beginning of shear and at a $K_{0}$ consolidation. Therefore, $k=1-K_{0}$ cannot be applied during shear. Moreover, in the "A" series tests of the discussed paper, the value of $\mathrm{K}_{0}$ changes during shear. When the value of $K_{0}$ is not constant during shear, the relation between $\frac{\tau}{\sigma_{v c}}$ and $\tan \psi$ becomes nonlinear if $k=1-K_{0}$. As a result, the use of the second method in the discussed paper does not provide an accurate prediction.

A method that can determine the coefficient $\mathrm{k}$ in a NGI-type simple shear apparatus is described by Budhu [4]. The value of $\mathrm{k}$ is determined from experimental results. The method assumes that at maximum stress ratio, the principal axes of stress and strain increment coincide. Recorded data shows the assumption holds true in simple shear [4,5,6,]. Adopting this method, the coefficient $\mathrm{k}$ can be calculated by Equation 1:

$k=\left|\frac{\tau}{\sigma_{v}} \cot \xi\right| \quad \operatorname{at}\left(\frac{\tau}{\sigma_{v}}\right)_{\max }$

Where: 
$\xi=$ rotation angle of principal axis of strain increment, $\xi$ can be calculated directly from boundary measurement.

\section{References}

[1] Oda, M. and Konishi, J., "Rotation of Principal Stresses in Granular Material During Simple," Soils and Foundations., Vol. 14, No. 4, 1974, pp. 39-53.

[2] Roscoe, K. H., Bassett, R. H., and Cole, E. R. L., "Principal Axes Observed During Simple Shear of a Sand," Proceedings of the Geotechnical Conference on Shear Strength Properties of Natural Soils and Rocks, Vol. 1, Norwegian Geotechnical Institute, Oslo, 1967, pp. 231-237.

[3] Stroud, M. A., 1971, "The Behavior of Sand at Low Stress Levels in the Simple Shear Apparatus," Ph.D. Dissertation., Cambridge University, Cambridge, United Kingdom.

[4] Budhu, M., 1979, "Simple Shear Deformation of Sands," Ph.D. dissertation., Cambridge University, Cambridge, United Kingdom.

[5] Wood, D. M., Drescher, A. and Budhu, M., "On the Determination of Stress State in the Simple Shear Apparatus,” Geotechnical Testing Journal., Vol. 2, No. 4, 1979, pp.211-221.

[6] Cole, E. R. L., 1967, "The behaviour of soils in the simple shear apparatus," Ph.D. dissertation., Cambridge University, Cambridge, United Kingdom. 